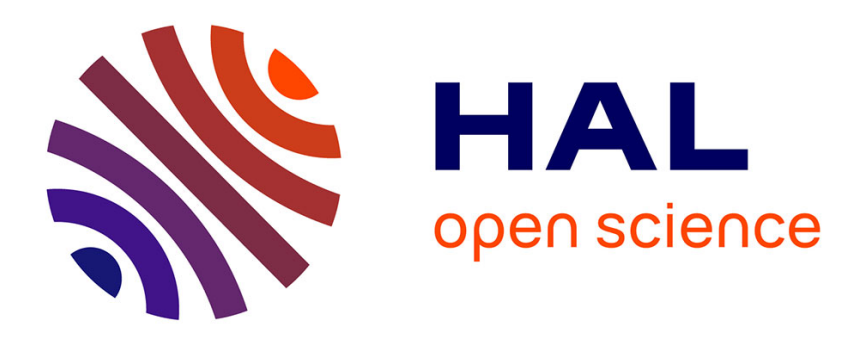

\title{
De l'appréhension des significations implicites : les syllogismes tronqués
}

Edith Salès-Wuillemin

\section{To cite this version:}

Edith Salès-Wuillemin. De l'appréhension des significations implicites: les syllogismes tronqués. Annee Psychologique, 1993, 93 (4), pp.345-378. halshs-00596048

\section{HAL Id: halshs-00596048 \\ https://shs.hal.science/halshs-00596048}

Submitted on 26 May 2011

HAL is a multi-disciplinary open access archive for the deposit and dissemination of scientific research documents, whether they are published or not. The documents may come from teaching and research institutions in France or abroad, or from public or private research centers.
L'archive ouverte pluridisciplinaire HAL, est destinée au dépôt et à la diffusion de documents scientifiques de niveau recherche, publiés ou non, émanant des établissements d'enseignement et de recherche français ou étrangers, des laboratoires publics ou privés. 


\title{
Persée
}

http://www.persee.fr

\section{De l'appréhension des significations implicites : les syllogismes tronqués}

\author{
E. Sales-Wuillemin \\ L'Année psychologique, Année 1993, Volume 93, Numéro 3 \\ p. 345 - 378
}

Voir l'article en ligne

Résumé De nombreuses études, tant en linguistique qu'en psycholinguistique, ont porté sur les mécanismes inférentiels mis en œuvre par les sujets afin de mettre au jour des informations non contenues explicitement dans des énoncés. II semble que cette recherche se fonde sur la cohérence interne des énoncés. Nous analysons le rôle joué par les connecteurs dans la mise en œuvre de ces mécanismes. Nous faisons l'hypothèse que ces processus reposent non seulement sur la cohérence argumentative des propositions mais également sur les savoirs activés par les sujets. Les instructions du connecteur venant renforcer (ou au contraire déstabiliser) cette cohérence. Deux expérimentations relatives au traitement de phrases complexes (correspondant à des syllogismes incomplets dans lesquels on a supprimé la prémisse majeure) sont présentées ici. Ces syllogismes incomplets ont été testés en faisant varier le connecteur reliant la prémisse mineure et la conclusion et l'orientation argumentative de ces deux propositions (qui pouvaient être coorientées ou anti-orientées). Les résultats mettent en évidence le fait que les deux facteurs (cohérence argumentative et instructions du connecteur) sont pris en compte. Toutefois, l'un peut être privilégié aux dépens de l'autre, cela dépend de la cohérence des énoncés. Nous avons ainsi pu montrer que lorsque les items sont cohérents, les sujets appliquent un traitement automatique, ce qui les conduit à privilégier le contenu des énoncés et à négliger les instructions véhiculées par le joncteur. Par contre, lorsque les items sont non cohérents, on assiste à deux phénomènes différents qui sont dus à une recherche de plausibilité. Soit le sujet privilégie le contenu et ignore les instructions du joncteur, soit il prend en compte ces instructions et rétablit la cohérence en imaginant un contexte explicatif. Le choix de l'une ou l'autre de ces stratégies dépend de la force du connecteur. Un énoncé contenant le connecteur «mais " peut ainsi recevoir deux lectures différentes : quand les items sont cohérents (c'est-à-dire lorsque les deux propositions sont anti-orientées) il peut exprimer un rejet de l'univers proposé au départ, par contre lorsque les items sont non cohérents (propositions co-orientées) il peut exprimer l'introduction d'une justification/explication de cet univers. A l'opposé, le connecteur «Pourtant » impose des contraintes interprétatives plus fortes, il est ainsi toujours interprété comme introduisant une opposition et ce même, si les contenus des deux propositions qu'il relie sont co-orientées. Mots clefs : inférence, signification implicite, connecteurs, compréhension du langage, cohérence.

\section{Avertissement}

L'éditeur du site «PERSEE »- le Ministère de la jeunesse, de l'éducation nationale et de la recherche, Direction de l'enseignement supérieur, Sous-direction des bibliothèques et de la documentation - détient la propriété intellectuelle et les droits d'exploitation. A ce titre il est titulaire des droits d'auteur et du droit sui generis du producteur de bases de données sur ce site conformément à la loi n98-536 du 1 er juillet 1998 relative aux bases de données.

Les oeuvres reproduites sur le site «PERSEE » sont protégées par les dispositions générales du Code de la propriété intellectuelle. 
Pour un usage strictement privé, la simple reproduction du contenu de ce site est libre.

Pour un usage scientifique ou pédagogique, à des fins de recherches, d'enseignement ou de communication excluant toute exploitation commerciale, la reproduction et la communication au public du contenu de ce site sont autorisées, sous réserve que celles-ci servent d'illustration, ne soient pas substantielles et ne soient pas expressément limitées (plans ou photographies). La mention Le Ministère de la jeunesse, de l'éducation nationale et de la recherche, Direction de l'enseignement supérieur, Sous-direction des bibliothèques et de la documentation sur chaque reproduction tirée du site est obligatoire ainsi que le nom de la revue et- lorsqu'ils sont indiqués - le nom de l'auteur et la référence du document reproduit.

Toute autre reproduction ou communication au public, intégrale ou substantielle du contenu de ce site, par quelque procédé que ce soit, de l'éditeur original de l'oeuvre, de l'auteur et de ses ayants droit.

La reproduction et l'exploitation des photographies et des plans, y compris à des fins commerciales, doivent être autorisés par l'éditeur du site, Le Ministère de la jeunesse, de l'éducation nationale et de la recherche, Direction de l'enseignement supérieur, Sous-direction des bibliothèques et de la documentation (voir http://www.sup.adc.education.fr/bib/ ). La source et les crédits devront toujours être mentionnés. 


\title{
MEMOIRES ORIGINAUX
}

\author{
Groupe de Recherche sur la parole \\ Université Paris VIII'
}

\section{DE L'APPREHENSION DES SIGNIFICATIONS IMPLICITES : LES SYLLOGISMES TRONQUES}

\author{
par Edith Sales-Wuillemin²
}

SUMMARY : Understanding implicit meanings : Incomplete syllogisms.

Various linguistic and psycholinguistic studies have been realized concerning processes activated by subjects in order to grasp informations not explicitly contained in statements. These processes seem to be based on the search of coherence.

We examine here how connectives can remove ambiguity. Our hypothesis is that these processes do not only depend on argumentative coherence of propositions but also on the subjects' knowledge. Connective instructions can reinforce, or on the contrary obscure, this coherence.

$T$ wo experiments on the processing of isolated complex sentences (incomplete syllogisms without major premiss) are presented here. These incomplete syllogisms were presented varying the connective between minor premiss and conclusion and the argumentative orientation of these two propositions (which could be co-orientated or anti-orientated).

Results show that both argumentative coherence and connective instructions interfer. Howpever one may prevail over the other, it depends on item coherence.

Thus we show that when items are coherent, subjects use an " automatic data processing "and they do not take into account connective instructions. On the contrary, when items are not coherent, subjects try to find a plausible statement. Two strategies are applied, subjects take into account connective

1. UFR 7 (PPGs), 2, rue de la Liberté, 93526 Saint-Denis Cedex 02.

2. Nous tenons à remercier E. Friemel, maitre de conférences à l'Université de Paris VIII, pour les conseils qu'il nous a donnés en ce qui concerne les traitements statistiques. 
instructions and re-establish coherence or they ignore these instructions. It depends on connective strength.

The French connective "Mais" (" but") can thus be interpreted in two different ways : when items are not coherent (when the two propositions are co-orientated), it can express either a rejection of the universe proposed in the first proposition or a justification/explanation of the universe proposed.

Contrary, the French connective "Cependant" (" however ") has greater strength, so that it is always interpreted as introducing an opposition, even if the two propositions are co-orientated.

Key-words : inference, implicit meaning, connectives, language comprehension, coherence.

\section{INTRODUGTION}

\section{OвJET}

Dans cet article, nous analyserons la façon dont les sujets mettent au jour des significations implicites véhiculées par des enthymèmes (syllogismes incomplets). Les enthytèmes renvoient à une catégorie d'implicite, les " lacunes dans l'enchaînement des énoncés ", qui se présente sous la forme d'une suite de propositions laissée incomplète et qui est généralement utilisée de façon stratégique. En effet, elle permet au locuteur d'exprimer un point de vue ou un état de fait sans porter la responsabilité de son dire, du fait même qu'il laisse à l'interlocuteur le soin de compléter l'enchaînement.

Nous nous intéressons dans ce contexte au rôle joué par des marques spécifiques, les joncteurs ${ }^{3}$, dans la mise au jour des implicites. Il semble en effet que la fonction de ces morphèmes ne se limite pas à l'articulation des propositions, ils jouent également un rôle dans la régulation des mécanismes inférentiels.

Après avoir introduit dans un premier temps quelques recherches qui portent sur le fonctionnement des enthymèmes, nous analyserons le rôle des joncteurs dans l'activation des inférences.

3. Nous utilisons le terme * joncteur s de prélérence à celui de * connecteur parce qu'il concrétise mieux, à notre sens, le rôle joué par ces morphèmes, à savoir marquer une relation de cohésion entre les propositions et donc, au-delà, d'assurer la cohérence des énoncés. Ce terme est emprunté à Ghiglione, Matalon et Bacri (1985). 
Nous présenterons dans un deuxième temps deux expérimentations. La première nous permettra de mesurer l'acceptation globale d'inférences tirées à partir d'enthymèmes dans lesquels on a fait varier le joncteur, la deuxième nous conduira à rendre compte des inférences réalisées (" spontanément ") par les sujets à partir de ces enthymèmes.

\section{Gadre théorique}

Notons tout d'abord que l'implicite relatif aux enthymèmes a été décrit entre autres par Ducrot (1972), et Kerbrat-Orecchioni (1986). Ici le locuteur laisse inexprimée soit l'une des prémisses (la majeure ou la mineure), soit la conclusion : à charge pour l'interlocuteur de reconstituer l'énoncé afin de rétablir la cohérence interne de celui-ci.

Pour décrire cet implicite, Ducrot (op. cit., p. 7) propose un exemple dans lequel la prémisse majeure est supprimée : “X. est venu me voir, il a donc des ennuis. "Ici l'interlocuteur doit nécessairement, selon Ducrot, inférer : "X. ne vient me voir que lorsqu'il a des ennuis. "L'analyse de Ducrot introduit done un raisonnement inductif qui se fonde sur la prémisse mineure et la conclusion. Cependant, le mécanisme inférentiel aboutit, dans cet exemple, à une forme " distordue " (la prémisse majeure ayant la forme d'une biconditionnelle (ou tout du moins celle d'une converse) et non, comme c'est le cas dans la forme canonique, celle d'une implication).

Kerbrat-Orecchioni (op. cit., p. 166-167) déclinera quant à elle cette recomposition du syllogisme en donnant une série d'exemples dans lesquels :

1) la prémisse mineure reste implicite (e.g. " C'est parce que je t'aime, que tu m'aimes quand même. Tu m'aimes pour mon amour, donc tu m'aimeras toujours ", où l'interlocuteur infère : "Or, je t'aimerai toujours ");

2) la conclusion reste implicite (e.g. "En ton absence je me suis aperçue d'une chose. J'aime les hommes de mon âge. Toi tu auras bientôt cinquante ans ", où l'interlocuteur infère : " Donc je ne t'aime pas ") ;

3) la prémisse majeure et la conclusion restent implicites (e.g. A. - "Voulez-vous un verre de Martini ?"B. - "Je suis musulman ", où l'interlocuteur infère : "Quand on est musulman, on ne boit pas d'alcool ", "donc B ne prendra pas d'alcool "); 
4) la prémisse mineure et la conclusion restent implicites (" La vie est trop courte pour s'habiller triste " où l'interlocuteur infère : "Or porter des Newman c'est s'habiller "pas triste" ", "Donc il faut porter des Newman ").

Toutefois, comme le souligne Kerbrat-Orecchioni, cette catégorie d'implicite ne renferme pas uniquement des syllogismes incomplets. En effet, elle repose plus généralement sur les relations de cohérence entre les différentes propositions explicites. Les énoncés concernés peuvent ainsi se présenter sous la forme de deux propositions reliées par un joncteur comme dans " $\mathrm{P}$ mais $Q$ ». Ici la proposition qui permet de relier $P$ et $Q$ n'est pas donnée explicitement, bien qu'elle soit à la base de l'enchaînement.

Ainsi l'énoncé : "Il avait vu Jeanne, mais il persistait néanmoins à vouloir l'épouser ", nécessite pour être compris l'élaboration d'une proposition intermédiaire qui pourrait être : "Or elle n'était guère épousable. "Ce type d'implicite repose donc selon l'auteur" sur l'utilisation du joncteur "mais " (ou de ses équivalents) qui permet de générer la proposition implicite.

Au terme de cette première analyse, différentes remarques doivent être faites. Elles concernent d'une part la nature des compétences mises en jeu pour découvrir l'implicite et d'autre part le rôle des joncteurs.

Concernant le premier point, on notera que les exemples cités ont des niveaux de complexité différents. En effet, alors que dans les deux premiers enthymèmes (décrits respectivement par Ducrot et Kerbrat-Orecchioni) la mise au jour de l'implicite ne repose finalement que sur le contenu des énoncés et sur les compétences logiques du sujet interprétant, dans les autres enthymèmes, l'inférence se fonde également (à des degrés différents) sur un ensemble de savoirs (compétences encyclopédiques), sur le contexte ainsi que sur l'application de règles conversationnelles. On pourrait ainsi, à partir des mêmes énoncés, imaginer d'autres contextes, c'est-à-dire faire appel à d'autres schémas (au sens de Minsky, 1975) ou scripts (Schank et Abelson, 1977) et aboutir à des conclusions très différentes.

Pour décrire ces implicites, on ne peut donc se fonder unique-

4. On pourrait ajouter à cela le fait que le connecteur « mais " n'est pas le seul responsable du déclenchement de l'implicite, entrent également ici en ligne de compte : 1) le verbe " persister * qui véhicule l'idée de «'obstiner malgré tout "; et 2) la conjonction "néanmoins " qui joue ici un rôle de renforçateur des instructions données pas le * mais". 
ment sur la dynamique du syllogisme, il est également nécessaire de faire appel aux savoirs du sujet interprétant, qui est "invité " à évoquer ou à rechercher en mémoire un contexte qui lui permette de rétablir la proposition implicite et donc la cohérence de l'énoncé. Dans ce processus, les joncteurs jouent un rôle fondamental, car ils déclenchent et régulent le mécanisme inférentiel.

Plus précisément, les joncteurs ont une fonctionnalité qui s'exerce à deux niveaux complémentaires : outre le fait qu'ils coordonnent des énoncés, ils permettent également :

1) de signaler l'existence d'une proposition implicite;

2) de marquer l'orientation argumentative de la (ou des) proposition(s) manquante(s) (l'orientation argumentative de cette dernière étant directement liée à la nature du joncteur utilisé ainsi qu'à l'orientation argumentative des propositions explicites).

Afin d'analyser plus avant la fonction des joncteurs nous envisagerons deux types de recherches qui se situent, d'une part, dans le champ pragmatique et conversationnel et, d'autre part, dans le cadre de la psycholinguistique. Les premières mettent l'accent sur les instructions procédurales véhiculées par les joncteurs, les secondes se focalisent sur le rôle joué par ces marques dans les activités de compréhension et de mémorisation de textes ou d'énoncés ${ }^{5}$.

\subsection{L'étude des connecteurs pragmatiques}

Les analyses pragmatiques vont être marquées par une évolution. En effet, on note que si au départ on a cherché à établir des catégories homogènes à partir de la fonction des joncteurs ${ }^{\mathbb{6}}$,

5. Nous ne prétendons pas ici à une exhaustivité concernant (l'abondante) littérature pragmalinguistique, conversationnelle ou psycholinguistique ayant trait aux joncteurs. Le lecteur pourra se reporter notamment aux ouvrages de Jayez (1988), Roulet, Auchlin, Moeschler, Rubattel et Schelling (1987), et Caron (1983), ainsi qu'aux articles de Moeschler (1989), Moeschler, Reboul, Luscher et Jayez (1991), Luscher (1989) pour les aspects pragmatiques ou de Kail et Weissenborn (1984) pour ce qui concerne leur acquisition.

6. Les * connecteurs pragmatiques " sont classés (cf. de Spengler, 1980) en differentes catégories, compte tenu de leur fonction. On distingue, entre autres, les connecteurs qui marquent la concession (mais, pourtant, bien que...), ceux qui marquent l'opposition (en revanche, par contre...), ceux qui introduisent une justiflcation/explication (car, parce que, puisque...), ou une conclusion (donc, aussi...), etc. 
on s'est rapidement aperçu de la difficulté à mettre au jour des propriétés spécifiques et invariables concernant les éléments de chaque catégorie. En effet, ces catégories n'étaient pas exclusives, certains joncteurs étant plurifonctionnels cela revenait à les classer dans l'une ou l'autre de ces catégories en fonction de leur contexte d'apparition. Dès lors, les auteurs ont cherché à rendre compte de la polysémie des joncteurs en mettant en évidence les instructions qu'ils véhiculent et ce en établissant des " schémas procéduraux " pour chacun d'eux (cf. Moeschler et al., 1991, op. cit.) et en introduisant la notion de force de connexion (cf. Luscher, 1989). Les relations entre les différents joncteurs sont donc analysées en termes de " proximité " et non plus seulement en faisant référence à des catégories. Dans ce contexte, l'éventail d'instructions véhiculé par un joncteur commande (avec plus ou moins de contraintes) le type d'inférences que l'allocutaire doit réaliser.

C'est ainsi qu'on s'accorde à dire, à la suite des travaux de Ducrot (1980), Ducrot et al. (1980), et de Anscombre et Ducrot (1977, 1983), que le joncteur "mais " possède une polysémie naturelle, car il a des contraintes interprétatives qui varient en fonction de l'orientation argumentative des propositions qu'il relie.

Ces deux auteurs (op. cit., 1977) ont ainsi pu montrer que ce joncteur peut avoir deux emplois différents :

- Le premier correspond à une réfutation directe. Dans ce cas, le " mais " peut être remplacé par " au contraire " comme dans :

(1) "Il n'est pas Français mais Allemand ".

- Le second correspond a un emploi argumentatif (opposition indirecte) : le " mais " reliant les deux propositions $p$ et $q$ permet :

1) de marquer une orientation de $p$ vers une conclusion possible $(r)$ et une orientation de $q$ vers une conclusion opposée (nonr);

2) d'attribuer à $q$ une force argumentative supérieure à celle de $p$. Ainsi par exemple l'énoncé :

(2) "Il est intelligent mais il ne travaille pas "

où la conclusion $r$ tirée à partir de la proposition $p$ peut être : "Il faut l'engager ", mais où $q$ oriente argumentativement vers la conclusion opporée, non- $r$ : "Il ne faut pas l'engager." 
Dans cette deuxième forme d'emploi du " mais " les propositions $p$ et $q$ ne s'opposent pas directement mais par l'intermédiaire de leurs conclusions respectives. On remarque que le joncteur " mais " peut être ici remplacé par " en revanche " ou par "par contre $n$.

Un cas particulier d'opposition plus directe peut toutefois se présenter : il existe des énoncés dans lesquels la conclusion $r$ que l'on peut tirer de $p$ correspond à la négation de $q$.

e.g. (3) "Il est républicain mais honnête ",

où $p$ oriente vers la conclusion $r$ : "Il n'est pas honnête. "

Ce cas se démarque des utilisations décrites ci-dessus de par le fait que l'on peut expliciter le "mais " par " pourtant ", " cependant " ou " néanmoins ". Ainsi, le joncteur "mais " possède une polysémie que n'a pas, par exemple, le " pourtant " qui est un oppositif direct (cf. à ce sujet Jayez, 1981, p. 185).

Luscher (1988) précisera cette analyse en distinguant d'une part les " mais " (qu'il classe dans les " connecteurs pragmatiques ") qui relient des arguments anti-orientés (les deux propositions s'opposant directement ou par l'intermédiaire de leurs conclusions respectives, comme dans les exemples (2) et (3)), et d'autre part les "mais" (qu'il classe dans les "opérateurs sémantiques") qui relient des arguments co-orientés (l'argument $q$ allant dans le même sens que la conclusion que l'on peut tirer de $p$ comme dans "il n'est pas pair mais impair " ou dans (1)).

De la même manière, si nous examinons les conjonctions causales " car ", " parce que " et " puisque ", on note (notamment à la suite des travaux de Ducrot $(1972,1983)$. Anscombre et Ducrot (1983), et de Barbault, Ducrot, Dufour, Espagnon, Israel et Manesse (1975)) que le "parce que " a un statut particulier. En effet, il introduit " à partir de deux idées $P$ et $Q$ qu'il relie une idée nouvelle, à savoir l'idée d'une relation de causalité entre $P$ et $Q$ ». "Car " et " puisque " sont utilisés au contraire pour " accomplir un acte de parole (acte de justification, d'inférence) : ils marquent que le locuteur effectue, à l'occasion et au moyen de ces idées, une activité de parole particulière " (p. 254).

De Fornel (1989) met quant à lui en évidence une utilisation particulière du " parce que " qui ne respecte pas la relation causale décrite traditionnellement. Il s'agit d'un " parce que " de conséquence qui fonctionne comme un " donc ". Le connecteur " puisque " permet quant à lui : 1) d'introduire un argument qui vient 
appuyer l'acte d'énonciation ; 2) de signaler que l'acte introduit a une valeur de rappel ; et 3) d'opérer un jeu sur la prise en charge des deux constituants, à savoir l'acte directeur et l'argument (cf. E. Roulet et al., 1987, op. cit., p. 130).

Enfin, pour ce qui concerne les joncteurs consécutifs comme " donc ", on note qu'ils permettent d'introduire une relation de conséquence nécessaire : face à un énoncé de la forme " $P$ donc $Q$ ", l'allocutaire est invité à évoquer un contexte dans lequel $P$ implique nécessairement $Q$. Plus précisément, " il y a non seulement, comme pour toutes ces marques, construction d'un contexte pour interpréter la phrase, mais exigence supplémentaire d'un contexte où $Q$ puisse apparaître nécessairement impliquée par $P$, (Jayez, 1988, op. cit., p. 39). Il est nécessaire toutefois d'introduire une distinction au sein de la catégorie des consécutifs. Cette distinction renvoie à la légitimité du processus de déduction. " Donc " se distingue de "en conséquence ", en ce sens qu'il fait référence à une norme (idéologique ou culturelle) qui est utilisée pour légitimer le processus de déduction. En revanche, l'emploie de " par conséquent " n'est acceptable que dans la mesure où ce calcul peut être attribué à l'énonciateur (cf. Roulet et al., 1987, op. cit., p. 148-149).

Après ce rapide tour d'horizon, il apparait que si ces recherches ont eu le mérite d'analyser précisément les instructions procédurales attachées aux connecteurs, elles ne rendent malheureusement pas toujours compte du rôle joué par ces marques dans l'organisation générale des textes, et au-delà de la façon dont elles influencent le processus de traitement. C'est de ces aspects que se chargeront les recherches psycholinguistiques.

\subsection{Les études psycholinguistiques}

Les différentes recherches réalisées dans le cadre de la psychologie et de la psycholinguistique ont pu mettre en évidence l'importance de ces morphèmes en ce qui concerne la compréhension et la mémorisation. On assistera là aussi à une évolution des conceptions.

En effet, si, comme le note Caron (1983), celles-ci se sont focalisées dans un premier temps sur des aspects logico-linguistiques et portaient principalement sur des énoncés hors contexte, actuellement on se préoccupe plus précisément $d u$ rôle de ces marques dans les activités de compréhension et de mémorisation en mettant l'accent sur leur fonction structurante, c'est-à-dire sur la 
cohérence du texte et au-delà sur l'activation des savoirs des sujets.

G'est ainsi que Caron-Pargue et Caron (1989) relèvent quatre fonctions essentielles des joncteurs. Ils permettent ainsi non seulement, comme nous l'avons vu, de véhiculer des instructions de traitement des énoncés et de moduler la prise en charge de ceux-ci, mais également de "délimiter les énoncés " pour leur stockage en mémoire et de repérer les informations qui feront l'objet d'enchâ̂nements ultérieurs.

De façon générale, Caron (1985) montre que l'emploi de joncteurs dans un texte favorise l'activité inférentielle et a une influence sur le stockage en mémoire.

Plus précisément, il semblerait que les joncteurs soient utilisés pour annoncer la nature d'un événement et donc son importance, ce qui déterminerait le stockage en mémoire. En somme, les joncteurs permettent d'organiser le discours et au-delà la représentation mentale qui est associée aux événements décrits. Ainsi, dans une épreuve de rappel indicé, Caron (1988) montre que les propositions reliées par le joncteur " et " sont rappelées séparément, alors que celles qui sont reliées par un " mais " apparaissent de façon conjointe ; ces résultats se retrouvant avec les joncteurs " parce que ", " mais » et " et " présentés dans des couples de phrases (Caron, Micko et Thüring 1988). De la même manière, Townsend (1983) constate que le segment introduit par " mais " est conservé dans sa forme littérale en mémoire et ce en vue d'un traitement ultérieur.

Enfin, on constate que certaines unités ont des " disponibilités " différentes en mémoire, ceci dépend du connecteur utilisé (cf. Caron, Caron-Pargue, Micko, Tomeh et Verdret 1987).

Un autre type de recherche se focalisera sur le rôle des connecteurs concernant l'organisation globale du texte et donc sur la cohérence de celui-ci. Une distinction est aujourd'hui opérée entre la cohésion et la cohérence. La cohésion est définie par Halliday et Hasan (1976) à partir des relations sémantiques explicites entre les phrases, elle rend donc compte de l'ordre d'apparition des différentes informations dans un texte ainsi que de l'enchaînement des phrases. La cohérence est une notion plus psychologique, car elle fait appel aux savoirs des sujets, en somme ce n'est pas une propriété du texte lui-même, mais une caractéristique attribuée au texte par les sujets et ce en fonction de leurs savoirs (Petöfi, 1983, p. 266). Les joncteurs interviennent à ces 
deux niveaux : ils ont une fonction de cohésion (en ce sens qu'ils marquent les enchainements entre les énoncés), mais ils jouent également un rôle en ce qui concerne la cohérence (en ce sens qu'ils activent des savoirs et structurent les représentations).

Dans une expérimentation réalisée sur des enfants, portant sur le rappel de récits, Mouchon, Fayol et Gombert (1989) ont ainsi pu montrer - à la suite des travaux de French et Nelson (1985) - que les joncteurs ont une fonction cohésive et que les enfants les introduisent spontanément tout en effectuant des choix en fonction de la structure du récit. De la même manière, partant du fait que la compréhension du discours nécessite la construction d'une représentation cohérente de ce discours et que pour élaborer cette représentation il est nécessaire d'élaborer des inférences concernant les relations de causes/conséquences et d'affirmations/arguments, Sanders et Spooren (1992) montrent que les joncteurs jouent un rôle important dans la mise au jour de cette cohérence et donc dans l'élaboration de cette représentation.

$\mathrm{Au}$ terme de cette analyse, on note que lors de la compréhension d'un texte (ou d'un discours) les joncteurs servent non seulement de moteur d'inférence (ils activent les savoirs des sujets), mais constituent également la trame du discours (ils marquent les relations de cohérence qu'entretiennent les énoncés et structurent la representation de ce discours\}.

Dans ce cadre, il est intéressant d'analyser la façon dont le sujet relie, grâce au mécanisme inférentiel, les marques linguistiques et les informations résidant en mémoire et plus généralement dans ce que l'on nomme le " contexte".

Une hypothèse est aujourd'hui admise concernant cet aspect : il semblerait en effet que ces inférences se fondent sur l'intention attribuée à la source, visent une " explication plausible (Bach et Harnisch, 1979, p. 92) et reposent sur la pertinence attribuée à l'énoncé (Sperber et Wilson, 1986).

\section{HYPothìsES}

Nous partirons du fait que l'emploi d'un joncteur concessif permet au locuteur de signaler à l'allocutaire son intention de lui voir inférer un contexte plausible qui puisse expliquer l'opposition, qu'il marque linguistiquement par le joncteur, entre les propositions données explicitement. L'emploi d'un joncteur de 
conséquence ou à valeur causale lui permet par contre de signaler son intention que l'allocutaire infère un contexte plausible qui puisse expliquer la co-orientation des propositions données explicitement.

Cependant, si la fonction du joncteur est un facteur important dans le parcours inférentiel de l'allocutaire, il est également nécessaire de prendre en compte l'orientation argumentative de chacune des propositions explicites (et au-delà les savoirs des sujets interprétants) ainsi que les relations qu'elles entretiennent, c'est-à-dire la cohérence globale de l'énoncé.

Ces remarques concernant les instructions de traitement véhiculées par un joncteur et la cohérence des énoncés dans lesquels il apparaît nous amènent à poser les hypothèses générales suivantes.

\section{HYPOTHESES}

H1 : lorsque les propositions explicites sont co-orientées et qu'elles sont reliées par un joncteur de cause ou de conséquence, celui-ci devrait renforcer la cohérence argumentative de l'énoncé, ce qui devrait maximiser les effets attendus par le locuteur. Le sujet devrait donc produire des inférences qui ont la forme d'une implication. Par contre, si elles sont reliées par un joncteur d'opposition, celui-ci devrait faire vaciller cette cohérence, ce qui devrait placer le sujet face à deux choix différents :

1) soit il privilégie l'orientation argumentative des propositions explicites (contenu propositionnel), auquel cas il devra : a) moduler la fonction du joncteur, ou $b$ ) omettre tout simplement de prendre en compte ce joncteur;

2) soit il prend en compte la valeur d'opposition du joncteur, auquel cas il devra imaginer un contexte qui permette de rétablir la cohérence.

H2 : Lorsque les propositions explicites sont anti-orientées, on devrait observer un phénomène inverse. Si les propositions sont reliées par un joncteur d'opposition, celui-ci devrait venir renforcer la cohérence globale de l'énoncé, ce qui devrait maximiser les effets attendus. Le sujet devrait ainsi produire des inférences qui ont la forme d'une opposition. Par contre, si elles sont reliées par un joncteur de conséquence ou à valeur causale, celui-ci 
devrait faire vaciller la cohérence ce qui devrait placer là aussi le sujet face aux deux choix décrits ci-dessus : 1) privilégier l'orientation argumentative des propositions explicites; ou 2) prendre en compte la valeur du joncteur et donc rétablir la cohérence.

Ces deux hypothèses générales doivent être spécifiées pour les cas de non-cohérence. En effet, le choix de l'une ou l'autre de ces deux stratégies n'est pas indépendant de la force du joncteur, c'est-à-dire des contraintes interprétatives qu'il impose. Ainsi, si le joncteur peut assumer différentes fonctions, le sujet devrait pouvoir adopter l'une ou l'autre des stratégies décrites ci-dessus, par contre si le joncteur possède des contraintes interprétatives fortes, le sujet devrait être amené à le prendre en compte et donc à rétablir la cohérence (deuxième stratégie).

Ces dernières réflexions nous amènent à poser les hypothèses spécifiques suivantes concernant les cas de non cohérence.

H3 : Lorsque des propositions co-orientées sont reliées par un joncteur d'opposition, on peut faire l'hypothèse que les sujets 1) privilégieront l'orientation argumentative des propositions lorsque ce joncteur est un " mais";

2) prendront en compte la valeur du joncteur lorsque celui-ci est un oppositif plus direct comme " pourtant " ou " cependant".

H4 : Par contre, lorsque des propositions anti-orientées sont reliées par un joncteur de cause ou de conséquence, on peut faire l'hypothèse que, ces joncteurs possédant des contraintes interprétatives relativement fortes, les sujets seront amenés à prendre en compte la valeur de ce joncteur.

\section{Construction du matériel expérimental}

Dans les deux expérimentations que nous présentons, le même matériel expérimental a été utilisé. Seules les modalités de réponse des sujets ont été modifiées.

\subsection{Les joncleurs testés}

Nous testons trois calégories de joncteurs. Chaque catégorie étant représentée par trois joncteurs, soit au total :

- 3 joncteurs de conséquence : " donc ", " en conséquence " et "par conséquent";

- 3 joncteurs à valeur causale : " car ", " parce que » et " puisque ";

- 3 joncteurs concessifs : " mais ", " pourtant " et " cependant". 


\subsection{Construction des items présentés aux sujets}

L'expérience présente une série de douze items. Ghaque item est composé d'un couple de propositions reliées systématiquement par l'un des joncteurs ci-dessus décrits. Ces propositions correspondent à la prémisse mineure et à la conclusion de douze syllogismes de base que nous avons construits.

Les items (syllogismes incomplets) proposés aux sujets ont la forme générale suivante :

$$
P_{m i} \quad J \quad r
$$

où $P_{m i}$ désigne la prémisse mineure,

$\mathrm{J}$ désigne le joncteur ;

et $\boldsymbol{r}$ désigne la conclusion.

Les 12 syllogismes de base ont pour forme initiale :

Prémisse majeure : "Chaque fois que $p$ est vraie, $q$ est vraie " (ce qui correspond à l'implication logique traduite en langue par l'expression : "Si $p$ alors $q$ ", et est symbolisée par l'expression " $p \rightarrow q \|)$.

Prémisse mineure : " $p$ est vraie".

Conclusion : " $q$ est vraie ".

Dans ces 12 syllogismes, 6 présentaient des faits neutres ou co-orientés, et les 6 autres des faits habituellement anti-orientés. Ainsi, les items tirés de ces syllogismes peuvent prendre deux formes : soit les deux propositions (prémisse mineure et conclusion) sont plutôt co-orientées (CO), soit elles sont anti-orientées (AO).

\section{Exemple :}

(L'ensemble des syllogismes construits se trouve en annexe.)

Item co-orienté :

Pierre a téléphoné à Jean (prémisse mineure).

Pierre a des ennuis (conclusion).

Item anti-orienté :

René est en retard (prémisse mineure).

René a un rendez-vous important (conclusion).

Ces items (enthymèmes) proposés aux sujets étaient construits en faisant varier systématiquement le type de joncteur qui reliait la prémisse mineure et la conclusion. 
Soient les items suivants :

Premier item co-orienté présenté :

Pierre a téléphoné à Jean DONC (EN CONSÉquence, PAR Conséquent) il a des ennuis.

Pierre a téléphoné à Jean car (Parce que, pursque) il a des ennuis.

Pierre a téléphoné à Jean mais (Pourtant, cependant) il a des ennuis.

Premier item anti-orienté présenté :

René est en retard DONC (EN CONSÉQUENCE, PAR CONSÉQUENT) il a un rendez-vous important.

René est en retard CAR (PARce gue, PUisque) il a un rendezvous important.

René est en retard mais (Pourtant, cependant) il a un rendez-vous important.

En résumé :

Pour les joncteurs de conséquence :

(items cohérents)

6 items (enthymèmes) co-orientés construits avec DONC;

6 avec PAR CONSÉQUENT ;

6 aVeC EN CONSÉQUENCE;

(items non cohérents)

6 items anti-orientés construits avec DoNC;

6 avec PAR CONSÉQUENT ;

6 aVeC EN CONSÉQUENCE.

Pour les joncteurs de cause :

(items cohérents)

6 items co-orientés construits avec CAR ;

6 avec PARCE QUE;

6 avec PUISQUE;

(items non cohérents)

6 items anti-orientés construits avec CAR ;

6 avec PARCE QUE;

6 avec PUISQue. 
Pour les joncteurs concessifs :

(items non cohérents)

6 items co-orientés construits avec MaIS ;

6 avec CEPENDANT;

6 avec Pourtant;

(items cohérents)

6 items anti-orientés construits avec maIs ;

6 avec CEPENDANT;

6 avec pourtant;

soit au total 108 items

\section{Première expérimentation}

Dans cette première expérimentation, les sujets devaient attribuer un degré de vérité/fausseté à une proposition inférable à partir de l'item présenté. Cette proposition correspondait à la prémisse majeure du syllogisme de base.

Trois groupes de 15 sujets ont été constitués, chaque groupe devant permettre de tester une catégorie de joncteur. Ainsi chaque sujet se voyait proposer 36 items ( 18 items co-orientés et 18 items anti-orientés) contenant :

- pour les sujets du groupe 1 : des joncteurs de conséquence (donc, par conséquent, en conséquence);

- pour les sujets du groupe 2 : des joncteurs de cause (car, parce que, puisque) ;

- pour les sujets du groupe 3 : des joncteurs concessifs (mais, pourtant, cependant).

\subsection{Plan expérimental}

\subsubsection{Les variables}

Variables indépendantes :

- 9 joncteurs répartis en trois catégories ;

- 36 items présentés dans un ordre aléatoire (18 items plutôt co-orientés et 18 items anti-orientés).

Variables dependantes :

— degré de vérité/fausseté accordé à la proposition inférence (prémisse majeure). 
Soit le plan expérimental suivant :

$$
\mathrm{S}_{15}<\mathrm{J3}>{ }^{*} \mathrm{C}_{2}
$$

où $\mathrm{S}$ désigne le nombre de sujets par groupe;

$\mathbf{J}$ désigne les trois catégories de joncteurs testés : 3 joncteurs de "conséquence " (groupe 1 ), 3 joncteurs de " cause " (groupe 2 ), 3 joncteurs " concessifs" (groupe 3 ). Chaque item étant présenté trois fois ;

G désigne la nature de l'item présenté (Items plutôt co-orientés Items anti-orientés).

\subsubsection{Population}

45 sujets volontaires ayant entre 20 et 25 ans, étudiants en premier cycle à l'Université, de langue maternelle française, ont été retenus.

\subsection{Procédure}

Chaque sujet recevait un livret expérimental constitué de 37 feuillets. Sur le premier de ces feuillets était inscrite la consigne suivante :

" Je vais vous présenter une série de phrases. Chacune de ces phrases sera suivie d'une proposition qui explicitera ou tirera une conséquence à partir des informations contenues dans la phrase présentée.

"Sous cette proposition se trouvera une échelle en 7 points allant de tout à fait faux à tout à fait vrai.

"Je vous demanderai d'entourer un des points de l'échelle, sachant que si vous entourez le 1 cela signifie que vous pensez que la proposition est tout à fait fausse, et que si vous entourez le 7 cela signifie que vous pensez que la proposition est tout à fait vraie. Les notes intermédiaires vous permettront de moduler votre jugement."

Exemple : " Pierre part à la campagne, en effet la météorologie a annoncé du beau temps."

Selon vous la phrase "Chaque fois que Pierre part à la campagne la métérotologie a annoncé du beau temps » est-elle vraie ou fausse ?

(Donnez votre réponse en entourant un des points de l'échelle.)

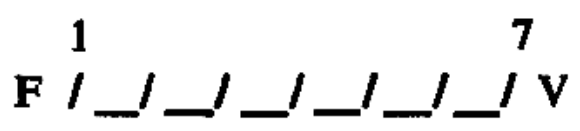


Après que les sujets eurent lu et compris la consigne, l'expérimentateur leur proposait de tourner la page. Chacun des 36 autres feuillets comportait :

1) Un item (composé d'une prémisse mineure et d'une conclusion reliées par un joncteur) dont la forme générale était

$P_{m l}$ Joncteur $r$.

2) Une proposition inférence correspondant à la prémisse majeure contenue dans les syllogismes construits au départ.

3) Une consigne demandant aux sujets d'attribuer un degré de vérité/fausseté à la proposition inférence, en entourant un des points d'une échelle.

\subsection{Analyse des résultats}

TableaU I. - Comparaisons intra-et inter-groupes (test du $T$ de Student). Les chiffres contenus dans les cases sont oblenus par simple moyennage des notes des sujets (se situant sur une échelle allant de 1 à 7), en distinguant les items co-orientes (CO) et les item anti-orientés (AO), quel que soit le joncteur

Within- and between-group comparisons ( $T$ test). The data in the cells are obtained by taking an average of subjects' scores on the attitude scale (ranging from 1 to 7). Here we distinguish co-orientated items (noted $\mathrm{CO}$ ) and Antiorientated items (noted AO)

\begin{tabular}{|c|c|c|c|}
\hline & 61 & 62 & 63 \\
\hline$C 0$ & $4,95 *$ & $3,54 *$ & 1,99 \\
\hline$A O$ & 4,31 & 3,38 & $1,65 *$ \\
\hline
\end{tabular}

G1 = Groupe 1 (Joncteurs de * conséquence $)$.

G2 $=$ Groupe 2 (Joncteurs de $*$ cause ).

G3 = Groupe 3 (Joncteurs * concessifs $*$ ).

Noté CO lorsque les items étaient co-orientés et AO lorsque les items étaient anti-orientés.

Noté $\leftrightarrow \cdots$ lorsque $p$ était inférieure ou égale à .05 .

Noté * lorsque les Items étaient cohérents.

Comparaisons intra-groupes :

Le résultat le plus marquant en ce qui concerne ce premier tableau réside dans le fait que les seuls joncteurs qui ne varient 
pas en fonction de l'orientation argumentative des propositions qu'ils relient (et donc en fonction de la cohérence de l'item) sont les joncteurs de cause testés dans le deuxième groupe $(t=1,38,14 \mathrm{dl}$, n.s. $)$.

Il semblerait donc que les contraintes interprétatives de ces joncteurs soient très fortes ce qui entraîne une prise en compte prépondérante de leur valeur. Ge résultat semble donc aller dans le sens de nos hypothèses. Il doit toutefois être modulé. En effet, on note que la prémisse majeure est toujours jugée plutôt fausse $(m=3,49)$. Il semblerait donc que si les joncteurs de cause instaurent une relation de cause à effet, cette relation n'est pas généralisée par les sujets qui ne la jugent pas applicable à toutes les occurrences de ces événements.

Si l'on effectue le même type de comparaison avec les joncteurs de conséquence (groupe 1), on observe, contrairement à nos hypothèses, une variation du degré de vérité/fausseté accordé à la prémisse majeure en fonction de l'orientation argumentative des propositions présentées. Lorsque ces propositions sont co-orientées (c'est-à-dire lorsque l'item est cohérent), la prémisse majeure est jugée globalement plus vraie que lorsque ces propositions sont anti-orientées, c'est-à-dire lorsque l'item est non cohérent $(t=2,91,14 \mathrm{dl}, p=.01)$. Il semblerait que les sujets ont jugé la vérité de la prémisse majeure en faisant référence à leurs savoirs, c'est-à-dire sur ce que l'on nomme "l'ordre normal des choses".

Pour ce qui concerne les joncteurs concessifs (groupe 3), on observe également une variation globale. La prémisse majeure est toujours jugée plus vraie lorsque les propositions explicites sont co-orientées $(t=2,22,14 \mathrm{dl}, p=.04)$, et ce alors même que le joncteur (de par ses instructions procédurales) vient déstabiliser ce lien (item non cohérent).

Incontestablement, là aussi jouent les savoirs des sujets, ce qui les conduit à privilégier l'orientation argumentative des propositions aux dépens des instructions véhiculées par le joncteur. Il semble donc bien que la première stratégie soit, dans ce dernier cas, privilégiée pour ce qui concerne les cas de non-cohérence.

Comparaisons inter-groupes :

Si l'on examine les comparaisons inter-groupes, on constate que l'effet du joncteur n'est pas pour autant annulé : la prémisse majeure est toujours jugée plus vraie lorsque les propositions 
explicites sont reliées par un joncteur de conséquence que lorsqu'elles sont reliées par un joncteur de cause ou un concessif et ce, que les items soient co-orientés ou anti-orientés (c'est-à-dire cohérents ou non cohérents). Les sujets semblent donc bien privilégier la première option de la première stratégie (option $a$ ) pour les cas de non-cohérence.

Tableau II. - Comparaisons intra-groupes (Test du $T$ de Student). Les chiffres contenus dans les cases sont obtenus par simple moyennage des notes données par les sujets. Sont regroupées ici les moyennes obtenues pour chaque joncteur

Within-group comparisons ( $T$ test). The data in the cells are obtained by taking an average of subjects' scores for the different connectives

\begin{tabular}{|c|c|c|c|c|c|c|}
\hline \multirow{2}{*}{ GROUPES } & CO* & AO & CO* & AO & CO & AO* \\
\hline$G 1$ & $4.48 *$ & 4.82 & $5.38 *$ & 4.73 & 5.89 & $4.19 *$ \\
\hline 62 & $3.29 *$ & 2.98 & $3.63 *$ & 3.56 & 3.69 & $3.34 *$ \\
\hline$G 3$ & $2.22 *$ & 1.65 & $1.54 *$ & 1.57 & 2.28 & $1.72 *$ \\
\hline
\end{tabular}

Groupe $1: \mathrm{J} 1=$ DONC - J2 = PAR CONSEQUENT - J3 = EN CONSÉQUENCE. Groupe 2: Jl = GAR - J2 $=$ PARCE QUE - J3 = PUISQUE.

Groupe $3: \mathfrak{J} 1=$ MAIS $-\mathrm{J} 2=$ POURTANT $-\mathfrak{J} 3=$ CEPENDANT.

Si on réalise des analyses locales, joncteur par joncteur, on note que les seuls joncteurs qui restent stables sont le "donc" qui marque la conséquence (G1-J1) et le " pourtant " qui marque la concession (G3-J2).

Il semble donc bien que ces deux joncteurs ont des contraintes interprétatives plus fortes que les autres joncteurs de leur catégorie ce qui conduit les sujets à privilégier les instructions qu'ils véhiculent (c'est-à-dire la deuxième stratégie décrite pour les cas de non-cohérence), et ce indépendamment de l'orientation argumentative des propositions qu'ils relient.

Le tableau III regroupe les résultats obtenus par moyennage au sein de chaque catégorie (tous joncteurs confondus). L'analyse de la variance montre une variation globale en fonction de la catégorie de joncteurs concernée $(\mathrm{F}(2,42)=28,53, p=.00001)$. Plus précisément on note que les joncteurs de conséquence signa- 
lent une co-orientation de la proposition manquante, les joncteurs concessifs marquent une anti-orientation. Ainsi, la prémisse majeure est jugée plutôt vraie $(m=4,95)$ lorsque la prémisse mineure et la conclusion sont reliées par un joncteur de conséquence ; et fausse $(m=1,82)$ lorsque ces deux propositions sont reliées par un joncteur concessif $(F(1,28)=59,14, p=0,0001)$.

TABleAU III. - Comparaison inter-groupe (analyse de la variance). Tableau global mettant en évidence les différences entre les groupes 1, 2 et 3 et plus précisément les différences entre les catégories de joncteurs (joncteurs de conséquence pour le groupe 1 , joncteurs de cause pour le groupe 2 et joncteurs d'opposition/concession pour le groupe 3)

Between-group comparisons (analysis of variance). This table displays differences between the three groups and more specifically between the different connectives (connectives introducing consequence (first group), cause (second group), and opposition (third group)

\begin{tabular}{|c|c|}
\hline GRQUPES/JONCTEUR5 & $\mathrm{J1}-\mathrm{J2}-\mathrm{J3}$ \\
\hline GROUPE 1 & $4.95 \mathrm{y}$ \\
\hline GROUPE 2 & $3.19 \mathrm{q}$ \\
\hline GROUPE 3 & $1.82 \mathrm{~d}$ \\
\hline
\end{tabular}

\subsection{Conclusion}

Cette première expérimentation nous a permis de mettre en évidence la fonction des joncteurs insérés dans des items au sein desquels nous avons fait varier l'orientation argumentative des propositions qu'ils reliaient. On note, à la fin de cette expérience que l'orientation argumentative des propositions explicites (et plus généralement les savoirs qu'elles mettent en jeu) est un facteur déterminant.

Ainsi, lorsque les sujets sont invités, à partir d'un énoncé, à puiser dans leur environnement cognitif et à vérifier l'adéquation entre cet environnement et la proposition faite, ils prennent en compte de façon privilégiée le contexte cognitif, les instructions véhiculées par le joncteur ne venant qu'en second lieu pour venir renforcer ou atténuer ce contexte. 
Ge résultat doit cependant être modulé en fonction de la particularité de certains joncteurs. Il semble en effet, au vu de cette expérimentation, que pour ce qui concerne les joncteurs de conséquence testés et la forme des items présentés, le joncteur " donc " véhicule des instructions plus contraignantes (et plus prégnantes) que les autres joncteurs de conséquence testés ("par conséquent" et "en conséquence »). Il en va de même en ce qui concerne le joncteur " pourtant " dans la catégorie des concessifs. Ainsi, pour ces deux joncteurs, l'orientation argumentative des propositions qu'ils relient n'a pas d'effet, seule est prise en compte la valeur du joncteur.

Ges résultats demandent toutefois à être complétés. En effet, cette expérimentation nous a permis de tester le degré de vérité attribué à une proposition inférable à partir d'un énoncé, proposition qui fonctionne à la manière d'un présupposé, mais rien ne nous garantit que cette proposition soit inférée "spontanément ".

En outre, cette technique de mesure ne nous permet pas d'appréhender de façon très fine les stratégies adoptées, et plus précisément si les sujets ont rétabli la cohérence et s'ils ont estimé que les items présentés étaient pertinents.

\section{DeuXième eXPÉrimentation}

Ici le principe est le même que dans la première expérimentation. Les mêmes items ont été utilisés. Toutefois la tâche des sujets ne consistait pas à attribuer un degré de vérité/fausseté à la prémisse majeure, il s'agissait pour eux de produire une inférence à partir de chaque syllogisme incomplet présenté.

\subsection{Population}

45 sujets volontaires ayant entre 20 et 25 ans, étudiants en premier cycle à l'Université, de langue maternelle française, ont été retenus.

\subsection{Procédure}

Comme dans la première expérimentation, chaque sujet recevait un livret expérimental contenant 37 feuillets. Sur le premier de ces feuillets était inscrite la consigne suivante :

" Je vais vous présenter une série de phrases. Pour chacune de ces phrases, je vous demanderai de donner en quelques lignes la ou 
les conclusions que vous tirez des informations contenues dans la phrase présentée."

Exemple :

"Pierre part à la campagne, en effet la météorologie a annoncé du beau temps."

Quelle(s) conclusion(s) tirez-vous de la phrase ci-dessous présentée ? .. . . . . . . . . . . . . . . . . .

\subsection{Codage et classement des réponses}

Les réponses des sujets ont été codées puis ventilées (en utilisant la méthode des juges) au sein de trois métacatégories que l'on a ensuite subdivisées en 8 catégories.

Ainsi pour ce qui concerne le premier syllogisme (cf. annexe) :

(Groupe 1) "Pierre a téléphoné à Jean, donc (en conséquence, par conséquent) il a des ennuis."

(Groupe 2) «Pierre a téléphoné à Jean, car (parce que, puisque) il a des ennuis. "

(Groupe 3) «Pierre a téléphoné à Jean, mais (pourtant, cependant) il a des ennuis."

Dans un premier temps ont été codées :

- I(mplication) les réponses qui établissaient une relation d'implication entre $p$ et $q$ ou entre $q$ et $p$.

- $O$ (pposition) les réponses qui établissaient une relation d'opposition entre $q$ et $p$.

- A(utre) les réponses qui ne rentraient pas dans les deux catégories ci-dessus définies.

Dans un deuxième temps, ces trois métacatégories ont été subdivisées, ce qui a abouti à la constitution de 8 catégories d'inférences.

Pour ce qui concerne les Implications ont été codées :

" $p \rightarrow q$ " les inférences de la forme : "Chaque fois que Pierre téléphone d Jean il en résulte des ennuis. "

" $q \rightarrow p$ " les inférences de la forme : "Chaque fois qu'il a des ennuis Pierre téléphone à Jean ", et les inférences de la forme : 
" En règle générale Pierre ne téléphone à Jean que lorsqu'il a des ennuis $\%$.

Pour ce qui concerne les Oppositions, ont été codées :

" $p \rightarrow \sim q$ " "Chaque fois que Pierre téléphone à Jean, il n'en résulte pas des ennuis " ou "Pierre n'a jamais d'ennuis après avoir téléphoné à Jean " ou encore "Pierre a eu des ennuis après avoir téléphoné à Jean ce qui est inhabituel ».

" $q \rightarrow^{\sim} p$ " "Chaque fois que Pierre a des ennuis, il ne téléphone pas à Jean" "Il ne téléphone jamais à Jean quand il a des ennuis" ou "Pierre a téléphoné à Jean bien qu'il ait des ennuis, contrairement à son habitude $n$.

Pour ce qui concerne les réponses Autres, ont été codées :

" $p \rightarrow q$ ou non- $q$ " les inférences de la forme : "Quand Pierre téléphone à Jean cela n'a pas forcément pour conséquence le fait qu'il ait des ennuis, il peut ou pas en avoir."

" $q \rightarrow p$ ou non- $p$ ": "Chaque fois que Pierre téléphone à Jean, il n'a pas forcément des ennuis, il peut ou pas en avoir."

A $(p . q)$ les inférences qui attestaient d'une co-occurrence entre les événements décrits dans $p$ et $q$ sans établir de relation de cause à effet (ex. : "Pierre avait des ennuis et il a téléphoné à Jean $")$.

"Autre cohérent" (Aco) les inférences qui, lorsque l'item était perçu à un premier degré comme étant non cohérent, visaient à rétablir à un second degré une cohérence entre $p$ et $q$. Etant donné qu'il n'y a pas eu d'inférences codées Aco pour ce premier syllogisme, nous exemplifierons à partir d'autres syllogismes. Ainsi pour le deuxième syllogisme (AO), ont été classées dans cette catégorie les inférences de la forme : "Benoit se couche tard pour réviser son examen ", ou pour le quatrième syllogisme (GO) : "La voiture de Ghristophe n'est pas en assez bon état pour faire un si long voyage."

\subsection{Analyse des résultats}

L'analyse des résultats fait apparaitre deux grands types de traitement qui sont relatifs à la cohérence des énoncés présentés et à un jeu sur les joncteurs.

Ainsi, pour les joncteurs consécutifs (G1) et les joncteurs de cause (G2), lorsque les énoncés sont cohérents, on assiste à un traitement quasi automatique des items, ce qui se traduit par des inférences qui ont la forme d'une implication, et ce quel que soit 
TABLEAU IV. - Dans ce tableau global figurent les fréquences d'occurrence (en pourcentages) des inférences produites par les sujets. Ces inférences ont été classées en trois catégories : Implications (noté " $I$ ), Oppositions (noté " $O$ ») et Autres (noté « $A$ ») puis réparties au sein des 8 catégories prédéfinies

This table contains occurrence frequencies (percentages) corresponding to inferences producted by subjects. These inferences were distributed amongst three categories : Implications (noted "I "), Oppositions (noted " $\mathrm{O}$ ) and Others (noted " $\mathrm{A}$ )) then we divided them amongst the eight categories defined above

\begin{tabular}{|c|c|c|c|c|c|c|}
\hline & & G1 & & G2 & & G3 \\
\hline & CO & NC & $\mathrm{CO}$ & NC & $\mathrm{CO}$ & NC \\
\hline \multicolumn{7}{|l|}{ Implications } \\
\hline$p \rightarrow q$ & 23.10 & 26.08 & 4.04 & 4.98 & 0.62 & 1.76 \\
\hline$q->p$ & 65 & 49.06 & 75.42 & 57.8 & 13.47 & 2.2 .64 \\
\hline Oppositions & & & & & & 1 \\
\hline$p->\sim q$ & 0 & 0 & 0 & $\mathbf{0}$ & 43.26 & 19.41 \\
\hline$q \rightarrow \sim p$ & 0 & 0 & 0 & 0 & 27.89 & 34.11 \\
\hline \multicolumn{7}{|l|}{ Autres } \\
\hline Aco & 8.2 & 24.8 & 8.08 & 31.89 & 7.83 & 22.05 \\
\hline$A(p \cdot q)$ & 3.3 & 0 & 10.43 & 5.31 & 0.3 & 0 \\
\hline$p->q$ ou $\sim q$ & 0 & 0 & 0.67 & 0 & 2.82 & 0 \\
\hline$q->p$ ou $\sim p$ & 0.3 & 0 & 1.34 & 0 & 3.44 & 0 \\
\hline Total & 1008 & $100 \%$ & $100 \%$ & $100 \%$ & 1008 & 1008 \\
\hline
\end{tabular}

Noté CO lorsque les items étaient cohérents et NC lorsque les items étaient non cohérents.

le joncteur considéré (G1, comparaison Implications/Oppositions/ Autres, $\mathrm{X} 2=1,44,4 \mathrm{dl}$, n.s. ; même comparaison pour G2, $\mathrm{X} 2=6,51,4 \mathrm{dl}$, n.s. $)^{2}$.

Conformément à nos hypothèses, le joncteur n'est pas ou peu

7. Etant donné que chaque sujet devait juger 36 items et que ces items étaient présentés dans un ordre aléatoire, on peut penser qu'il n'y a pas d'effet de répétition de la mesure $\mathrm{J} 1, \mathrm{~J} 2, \mathrm{~J} 3$, c'est pourquoi nous les avons traités comme des items indépendants. 
pris en compte. Tout se passe comme si celui-ci était considéré comme négligeable par rapport au contenu propositionnel et donc au schéma évoqué. Ceci se traduit par une forte proportion d'inférence de la forme $q \rightarrow p$ (plus de $65 \%$ des réponses) dans ces deux groupes expérimentaux, et ce même si le joncteur est censé marquer une relation de la forme $p \rightarrow q$ (joncteurs consécutifs).

Par contre, lorsque les items sont non cohérents, tout se passe comme si, le traitement automatique ne pouvant être appliqué, les sujets se référaient également aux instructions véhiculées par le joncteur. Le joncteur est alors en partie "rétabli " dans ses fonctions, ce qui se traduit par une légère diminution des inférences de la forme $q \rightarrow p$ lorsque ce joncteur est un consécutif, mais surtout par une très forte augmentation des inférences qui visent à rétablir la cohérence.

Ainsi, on note que dans les cas de non-cohérence les sujets de ces deux groupes produisent massivement des inférences qui visent à rétablir la cohérence (inférences que nous avons notées "Aco ") : G1 comparaison Items cohérents/non cohérents, Aco/Autres, $\mathrm{X} 2=27,1 \mathrm{dl}, p \rightarrow .001 ; \mathrm{G} 2$, même comparaison, $\mathrm{X} 2=38,68,1 \mathrm{dl}, p<.001\rangle$. Ces inférences se présentent sous la forme de contextes explicatifs qui visent à mettre l'accent sur le fait que l'énoncé est plausible malgré son incohérence apparente.

Pour ce qui concerne les joncteurs d'opposition (G3), on note également une apparition de ces deux grands types de traitement. Ainsi lorsque les items sont cohérents (c'est-à-dire lorsque ces joncteurs relient des propositions anti-orientées), les sujets appliquent là aussi un traitement automatique, ce qui les conduit à produire massivement des inférences qui ont la forme d'une opposition (71\% des inférences produites).

Quand les items sont non cohérents les sujets adoptent, conformément à nos hypothèses, deux stratégies différentes :

- Soit ils privilégient le joncteur et sont donc amenés à produire des inférences en forme d'opposition et à imaginer un contexte qui puisse expliquer cette apparente non-cohérence, ce qui se traduit par une augmentation des inférences qui visent à rétablir la cohérence (inférences notées "Aco ") : G3, comparaison Items cohérents/non cohérents, inférences Aco/Autres, $X 2=41,43$ $1 \mathrm{dl}, p<.001)$.

- Soit ils privilégient le contenu propositionnel auquel cas la valeur d'opposition du joncteur est modulée, ce qui les conduit à produire des inférences des deux formes $q \rightarrow p$ et Opposition. 
Tableau V. - Ce tableau met en évidence les résultats du troisième groupe de sujets (Groupe Opposition). Nous avons distingué ici 1) la cohérence des items; et 2) les trois connecteurs (J1 " mais », J2 " pourtant " et J3 " cependant ))

This table shows the results for the third group ("Opposition " group). We distinguished 1) coherence of items ; and 2) the three connectives ("But " noted J1, "However" noted J2 and "Yet " noted J3)

\begin{tabular}{||c||c|c|c|c|c|c||}
\hline & J1 & J2 & J3 & J1 & J2 & J3 \\
\hline \hline Implications & & & & & & \\
\hline p->q & 0.89 & 0 & 0.97 & 4.34 & 0.81 & 0 \\
\hline q->p & 25 & 5.76 & 8.83 & 49.56 & 1.62 & 17.64 \\
\hline Oppositions & & & & & & \\
\hline p-> q & 39.28 & 45.19 & 45.63 & 16.52 & 24.39 & 16.66 \\
\hline q-> p & 20.53 & 32.69 & 31.06 & 17.39 & 41.46 & 44.11 \\
\hline Autres & & & & & & \\
\hline Aco & 4.46 & 12.5 & 6.79 & 12.17 & 31.70 & 21.56 \\
\hline A (p.q) & 0.89 & 0 & 0 & 0 & 0 & 0 \\
\hline p->q ou $\sim q$ & 2.67 & 2.88 & 2.91 & 0 & 0 & 0 \\
\hline q->p ou p & 6.25 & 0.96 & 3.88 & 0 & 0 & 0 \\
\hline \hline Total & 1008 & 1008 & 1008 & 1008 & $100 \%$ & 1008 \\
\hline
\end{tabular}

Il semble bien que le choix de l'une ou de l'autre des stratégies soit dépendant de la force du joncteur.

Si l'on examine le tableau $\mathrm{V}$ on note que les sujets adoptent préférentiellement la première stratégie lorsque l'item contient le joncteur " pourtant ", et la seconde lorsqu'il s'agit d'un " mais " (et dans une moindre mesure d'un "cependant"). Ainsi, dans ce dernier cas, le joncteur est effectivement traité dans certains cas comme un joncteur de cause, ce qui se traduit par une forte proportion d'inférences de la forme $q \rightarrow p$ (49\% des réponses) : G3, Comparaison J1/J2/J3 Implications/Oppositions X $2=75,37$, $2 \mathrm{dl}$, p. $<.001)$.

Ainsi l'item "Pierre a mis son costume gris mais (cependant) il a un rendez-vous " est interprété comme " $\mathrm{P}$. a mis son costume 
gris mais (c'est parce que...) il a un rendez-vous " ou comme "P. a mis son costume gris car il a un rendez-vous".

A l'opposé, lorsque le joncteur est interprété comme introduisant une opposition, les sujets imaginent un contexte dans lequel " avoir son costume gris " et "aller à un rendez-vous " sont opposables, ce qui se traduit par des inférences de la forme : « Le costume de Pierre est démodé. "

\section{Discussion et conclusions}

Le résultat le plus marquant est relatif au jeu opéré entre la cohérence globale de l'énoncé traité et la force des joncteurs.

On note ainsi deux cas de figure : lorsque les items sont cohérents, c'est le contenu des énoncés qui prime, ce qui conduit les sujets a ignorer (ou moduler) les instructions véhiculées par le joncteur. On assiste donc à un traitement "économique " du système qui consiste à balayer l'item et à vérifier la cohérence argumentative des propositions. Dans ce contexte, le joncteur ne joue qu'un rôle second.

Par contre, lorsque les items sont non cohérents on voit se dessiner deux stratégies : soit le joncteur est pris en compte dans toute sa force, auquel cas les sujets sont amenés à produire un contexte plausible qui puisse rétablir la cohérence, soit le joncteur est modulé de façon à ce qu'il puisse se plier à cette utilisation atypique.

Le choix de l'une ou l'autre de ces stratégies est directement dépendant de la force du joncteur. Ainsi, si le joncteur a une force intrinsèque très grande (comme cela semble être le cas de " donc " et de "pourtant " dans les items testés), il est pris en compte ; par contre, s'il est d'une force intermédiaire (ou faible) alors on note qu'il a plus de chance d'être annulé ou modulé dans la mesure où il contrevient à la cohérence argumentative de l'énoncé.

Ce dernier résultat se concrétise à travers l'interprétation du " mais". En effet, nous avons noté qu'il pouvait générer des inférences qui peuvent prendre la forme d'une opposition et/ou d'une implication.

Ce résultat ne va toutefois pas à l'encontre des recherches effectuées antérieurement, comme par exemple celles de König (1985) qui attestent que les concessifs ne peuvent être interprétés comme marquant une autre relation. 
En effet, on peut constater ici que dans les deux cas, le " mais» a une valeur d'opposition. Toutefois, au lieu d'enchaîner en les réfutant sur les conséquences ou les conclusions que l'on pourrait tirer de $p$ (comme le décrivent Ducrot et Anscombre), il enchaîne (en les réfutant) sur les causes que l'on pourrait attribuer à $p$, ce qui se traduit dans les protocoles expérimentaux par une inférence de la forme $q \rightarrow q$.

Dès lors, on peut envisager deux grands types d'enchaînements indirects entre $p$ et $q$, que l'on peut croiser avec l'orientation argumentative de $p$ et $q$ : enchaînement sur les causes et enchaînement sur les conséquences ou les faits cooccurrents avec $p$.

Toutefois, on note alors que dans l'enchaînement sur les causes, $p$ et $q$ peuvent être co-orientées ou anti-orientées, dans l'enchaînement sur les conséquences, $p$ et $q$ doivent en principe être anti-orientées.

1) Enchaînement qui porte sur les causes de $p$.

Avec $p$ et $q$ co-orientés : "Il est alité mais il est malade."

Avec $p$ et $q$ anti-orientés : "Il est alité mais il n'est pas malade. "

2) Enchaînement qui porte sur les conséquences de $p$ (conclusions) ou sur les événements généralement cooccurrents avec $p$.

Avec $p$ et $q$ co-orientés (?) : "Il est alité mais il ne fera pas son travail."

Avec $p$ et $q$ anti-orientés : " Il est alité mais il fera son travail. »

En somme, construire un énoncé de la forme " $p$ mais $q$ " dans lequel " mais " introduit une opposition, c'est construire un univers de référence à partir de $p$ et $q$ dans lequel $q$ réduit l'ensemble des possibles générés par $p$, ou réfute un monde qui pourrait être généré par $\mathrm{p}$ ou à partir de $\mathrm{p}$.

On pourrait ainsi mettre en évidence une échelle polémique du " mais " qui se décomposerait comme suit :

1) "Mais " introduisant une réfutation qui porte directement sur $p$. Auquel cas $p$ et $q$ sont évoqués comme deux mondes incompatibles ;

2) "Mais " introduisant une réfutation qui porte sur les causes ou les conséquences de $p$. Auquel cas $p$ et $q$ sont compatibles et entretiennent des relations d'ordre logique.

3) " Mais " introduisant une précision (réduction) sur l'ensemble des possibles générables à partir de $p$. Auquel cas $p$ et $q$ sont totalement compatibles et entretiennent des relations d'inclusion. 


\section{ANNEXE}

\section{Construction des items}

\section{Liste des 6 syllogismes plutôt co-orientés}

1) Chaque tois que Pierre téléphone à Jean il a des ennuis (prémisse majeure)

Pierre a téléphoné à Jean (prémisse mineure)

Pierre a des ennuis (conelusion)

2) Chaque fois que Jean met son costume gris il a un rendez-vous (prémisse majeure)

Jean a mis son costume gris (prémisse mineure)

Jean a un rendez-vous (conclusion) majeure)

3) Chaque fois que Christophe prend sa voiture il va en province (prémisse

Christophe prend sa voiture (prémisse mineure)

Ghristophe va en province (conclusion)

4) Chaque fois que Christian lit des romans il est dans sa maison de campagne (prémisse majeure)

Christian lit des romans (prémisse mineure)

Christian est dans sa maison de campagne (conclusion)

5) Chaque fois que Serge prend l'avion il se rend à Toulouse (prémisse majeure)

Serge prend l'avion (prémisse mineure)

Serge se rend à Toulouse (conclusion)

6) Chaque fois que Pascal fait de l'équitation il est en vacances chez ses parents (prémisse majeure)

Pascal fait de l'équitation (prémisse mineure)

Pascal est en vacances chez ses parents (conclusion)

\section{Liste des 6 syllogismes anti-orientés}

1) Chaque fois que René est en retard il a un rendez-vous important (prémisse majeure)

René est en retard (prérnisse mineure)

René a un rendez-vous important (conclusion)

2) Chaque fois que Benoit se couche tard il a un examen (prémisse majeure)

Benoit se couche tard (prêmisse mineure)

Benoit a un examen (conclusion)

3) Chaque fois que Eric est fiévreux il fait beaucoup de sport (prémisse majeure)

Eric est flévreux (prémisse mineure)

Eric fait beaucoup de sport (conclusion)

4) Chaque fois que Fabrice mange du chocolat il est au régime (prémisse majeure)

Fabrice mange du chocolat (prémisse mineure)

Fabrice est au régime (conclusion) 
5) Chaque fois que Hervé part en vacances il a un travail urgent à faire (prémisse majeure)

Hervé part en vacances (prémisse mineure)

Hervé a un travail urgent à faire (coqclusion)

6) Chaque fois que Laurent boit du café le soir il doit se coucher tôt (prémisse majeure)

Laurent boit du café ce soir (prémisse mineure)

Laurent doit se coucher tôt (conclusion)

\section{TABLEAUX COMPLÉMENTAIRES}

TABleau VI. - Ce tableau concerne le groupe 1 (joncteurs de conséquence). Dans ce tableau figurent les inférences produites par les sujets et le nombre de réponses en pourcentage. Nous avons distingué : 1) la cohérence des items : items cohérents (CO) et items non cohérents (NC); et 2) les trois connecteurs : J1 (Donc), J2 (En conséquence), J3 (Par conséquent)

This table shows the results for the first group (connectives introducing consequence). This table displays inferences made by subjects and the number of responses in percent. We distinguished 1) coherence of items : coherent items (noted CO) and non coherent items (noted NG); and 2) the three connectives : J1 (Therefore), J2 (Consequently), J3 (In consequence)

\begin{tabular}{|c|c|c|c|c|c|c|}
\hline Inférences & J1 & $\begin{array}{c}\text { Items co } \\
\text { J2 }\end{array}$ & 53 & J1 & $\mid \begin{array}{c}\text { Items } \\
\mathrm{J} 2\end{array}$ & J3 \\
\hline $\begin{array}{c}\text { Implications } \\
\mathbf{p}->\mathrm{q}\end{array}$ & 15.31 & 30.2 & 25 & 21.05 & 29.34 & 28.43 \\
\hline $\mathbf{q} \rightarrow \mathbf{p}$ & 74.77 & 56.25 & 62.5 & 53.5 & 50.94 & 42,15 \\
\hline $\begin{array}{c}\text { Oppositions } \\
\text { p-> q }\end{array}$ & 0 & 0 & 0 & 0 & 0 & 0 \\
\hline$q \rightarrow>\sim p$ & 0 & 0 & 0 & 0 & 0 & o \\
\hline $\begin{array}{c}\text { Autres } \\
\text { Aco }\end{array}$ & 8.1 & 8.33 & 8.33 & 25,43 & 29.81 & 29.41 \\
\hline$A(p \cdot q)$ & .9 & 5.2 & 4.16 & 0 & o & o \\
\hline$p->q$ ou $-q$ & 0 & 0 & 0 & 0 & 0 & 0 \\
\hline$q->p$ ou $\sim p$ & 0.9 & 0 & 0 & 0 & 0 & 0 \\
\hline TOTAL & 100 & 100 & 100 & 100 & 100 & 100 \\
\hline
\end{tabular}


TABleaU VII. - Ce tableau concerne le groupe 2 (joncleurs de cause). Dans ce tableau figurent les inférences produites par les sujets et le nombre de réponses en pourcentage. Nous avons distingué : 1) la cohérence des items : items cohérents (CO) et items non cohérents (NC); et 2) les trois connecteurs : J1 (Car), J2 (Parce que), J3 (Puisque).

This table shows the results for the second group (connectives introducing cause). This table displays inferences made by subjects and the number of responses in percent. We distinguished 1) coherence of items : coherent items (noted $\mathrm{CO}$ ) and non coherent items (noted NC) ; and 2) the three connectives: J1 (For), J2 (Because), J3 (Since).

\begin{tabular}{|c|c|c|c|c|c|c|}
\hline Inferences & J1 & $\begin{array}{c}\text { Items co } \\
\text { J2 }\end{array}$ & J3 & J1 & \begin{tabular}{|c|} 
Items \\
J2
\end{tabular} & J3 \\
\hline $\begin{array}{c}\text { rmplications } \\
\text { p->q }\end{array}$ & 3.84 & 5.1 & 3.15 & 4.58 & 1 & 9.5 \\
\hline$q->p$ & 75.96 & 69,38 & 81.05 & 57.79 & 55.67 & 60 \\
\hline $\begin{array}{c}\text { Oppositions } \\
\text { p-> q }\end{array}$ & 0 & 0 & $\mathbf{0}$ & 0 & 0 & 0 \\
\hline$q->\sim p$ & 0 & 0 & 0 & 0 & 0 & 0 \\
\hline $\begin{array}{c}\text { Autres } \\
\text { Aco } \\
\end{array}$ & 6.73 & 11.22 & 6.31 & 44.3 & 39.17 & 24.21 \\
\hline$A(p, q)$ & 10.57 & 11.22 & 9.47 & 5.5 & 4.12 & 6.31 \\
\hline$p->q$ ou $\quad-q$ & 0.96 & 1.02 & $\mathbf{0}$ & 0 & 0 & 0 \\
\hline$q->p$ ou $\sim p$ & 1.92 & 2.04 & 0 & 0 & 0 & 0 \\
\hline TOTAL & 100 & 100 & 100 & 100 & 100 & 100 \\
\hline
\end{tabular}

\section{$R E S U M E$}

De nombreuses études, tant en linguistique qu'en psycholinguistique, ont porté sur les mécanismes inférentiels mis en cuvre par les sujets afin de mettre au jour des informations non contenues explicitement dans des énoncés. Il semble que cette recherche se fonde sur la cohérence interne des énoncés.

Nous analysons le rôle joué par les connecteurs dans la mise en ouvre de ces mécanismes. Nous faisons l'hypothèse que ces processus reposent non seulement sur la coherence argumentative des propositions mais également sur les savoirs activés par les sujets. Les instructions du connecteur venant renforcer (ou au contraire déstabiliser) cette cohérence. 
Deux experimentations relatives au traitement de phrases complexes (correspondant à des syllogismes incomplets dans lesquels on a supprimé la prémisse majeure) sont présentées ici. Ces syllogismes incomplets ont été testés en faisant varier le connecteur reliant la prémisse mineure et la conclusion et l'orientation argumentative de ces deux propositions (qui pouvaient étre coorientées ou anti-orientées).

Les résultats mettent en évidence le fait que les deux facteurs (cohérence argumentative et instructions du connecteur) sont pris en compte. Toutefois, l'un peut étre privilégié aux dépens de l'autre, cela dépend de la cohérence des enoncés.

Nous avons ainsi pu montrer que lorsque les items sont cohérents, les sujets appliquent un traitement automatique, ce qui les conduit à privilégier le contenu des énoncés et à négliger les instructions véhiculées par le joncteur. Par contre, lorsque les items sont non coherents, on assiste dे deux phenomènes différents qui sont dus à une recherche de plausibilité. Soit le sujet privilegie le contenu et ignore les instructions du joncteur, soit il prend en compte ces instructions et rétablit la coherence en imaginant un contexte explicatif. Le choix de l'une ou l'autre de ces stratégies dépend de la force du connecteur.

Un énoncé contenant le connecteur " mais" peut ainsi recevoir deux lectures différentes : quand les items sont cohérents (c'est-à-dire lorsque les deux propositions sont anti-orientées) il peut exprimer un rejet de l'univers proposé au départ, par contre lorsque les items sont non cohérents (propositions co-orientées) il peut exprimer l'introduction d'une justification/explication de cet univers. A l'opposé, le connecteur "Pourtant "impose des contraintes interprétatives plus fortes, il est ainsi toujours interprété comme introduisant une opposition et ce même, si les contenus des deux propositions $q u$ 'il relie sont co-orientées.

Mots clefs : inference, signification implicite, connecteurs, compréhension du langage, cohérence.

\section{BIBLIOGRAPHIE}

Anscombre J.-C., Ducrot O. - (1977) Deux Mais en français ?, Lingua, 43, 23-40.

Anscombre J.-C., Ducrot O. - (1983) L'argumentation dans la langue, Bruxelles, Mardaga.

Bach K., Harnish R. - (1979) Linguistic communication and speech acts, Cambridge, Mit Press.

Barbault M.-C., Ducrot O., Dufour J., Espagnon J., Israël C., Manesse D. (1975) Car, parce que, puisque, Reuue Romane, 10, 2, 248-280.

Caron J. - (1983) Les régulations du discours, psycholinguistique et pragmatique du langage, Paris, PUF.

Caron J. - (1985) Le role des marques argumentatives dans le rappel d'un texte, Bulletin de Psychologie, 38, 371, 775-783.

Caron J. - (1988) * Schémas de sens * et a effets de sens ": la sémantique des termes fonctionnels, Actes du Colloque Cognition et Connaissance : Ou va 
la science cognitive?, (Toulouse, mars 1988), Paris, Association pour la Recherche cognitive.

Caron J., Caron-Pargue J., Micko H. C., Tomeh B., Verdret P. - (1987) Analyse sémantique de connecteurs et de quantificateurs du langage naturel, Rapport final d'une recherche sous contrat CNRS (ATP 9.83.81).

Caron J., Micko H. C., Thüring M. - (1988) Conjunctions and the recall of composite sentences, Journal of Memory and Language, 27, 309-323.

Caron-Pargue J., Caron J. - (1989) Processus psycholinguistique et analyse des verbalisations dans une tache cognitive, Archives de Psychologie, 57, $220,3-32$.

Ducrot 0. - (1972) Dire et ne pas dire, Paris, Hermann.

Ducrot 0. - (1980) Analyses pragmatiques, Communications, 32, 11-60.

Ducrot O. et al. - (1980) Les mots du discours, Paris, Editions de Minuit.

Ducrot O. - (1983) Opérateurs discursifs et visée argumentative, Cahiers de Linguistique Française, 5, 7-36.

De Fornel M. - (1989) * Parce que ^ et le problème de l'inférence, Cahiers de Linguistique Française, 10, 171-192.

De Spengler N. - (1980) Première approche des marqueurs d'interactivité, Cahiers de Linguistique Frangaise, 1, 128-148.

French L. A., Nelson K. - (1985) Young children's knowledge of relational terms, New York, Berlin, Springer-Verlag.

Ghiglione R., Matalon B., Bacri N. - (1985) Les dires analysés : l'analyse propositionnelle du discours, Paris, Presses Universitaires de Vincennes.

Halliday M., Hasan R. - (1976) Cohesion in English, Londres, Longman.

Jayez J. - (1981) Etude des rapports entre l'argumentation et cerlains adverbes français, thèse de troisième cycle, Aix-Marseille III.

Jayez J. - (1988) L'inférence en langue naturelle, Paris, Hermès.

Kail M., Weissenborn J. - (1984) L'acquisicion des connecteurs : criliques et perspectives, in M. Moscato et G. Piéraut Le Bonniec (Edit.), Le langage, construction et actualisation, Rouen, Publications de l'Université de Rouen, $\mathrm{n}^{\circ}$ 98, 101-118.

Kerbrat-Orecchioni C. - (1986) L'implicite, Paris, A. Colin.

Konig E. - (1985) On the history of concessive connectives in English. Diachronic and synchronic evidence, Lingua, 66, 1-19.

Luscher J. M. - (1988) Signification par l'opérateur sémantique et inférence par le connecteur pragmatique, l'exemple de mais, Sigma, 12, 233-253.

Luscher J. M. - (1989) Connecteurs et marques de pertinence, l'exemple de d'ailleurs, Cahiers de Linguistique Française, 10, 101-145.

Minsky M. - (1975) A framework for representing knowledge, in P. Winston (Edil.), The Psychology of computer vision, New York, McGraw-Hill, 211-277.

Moeschler J. - (1989) Marques linguistiques, interprétation pragmatique et conversation, Cahiers de Linguistique Française, 10, 43-76.

Moeschler J., Reboul A., Luscher J. M., Jayez J. - (1991) Procédures interprétatives et savoirs partagés, Aix-en-Provence, Colloque du Iz au 14 seplembre 1991, "L'analyse des interactions ».

Mouchon S., Fayol M., Gombert J. E. - (1989) L'utilisation de quelques connecteurs dans des rappels de récits chez des enfants de 5 à 8 ans, L'Année Psychologique, 89, 513-529.

Petof J. S. - (1983) Text, signification, models and correlates, in G. Rickheit et M. Bock (Edit.), Psycholinguistic studies in language processing, Berlin, New York, W. de Gruyter.

Roulet E., Auchlin A., Moeschler J., Rubattel C., Schelling M. - (1987) $L$ 'articulation du discours en français contemporain, Berne, Peter Lang. 
Sanders T. J. M., Spooren W. P. M. - $(1992\rangle$ Toward a taxonomy of coherence relations, Discourse Processes, 15, 1-35.

Schank R. C., Abelson, R. P. - (1977) Scripts, plans, goals and undersanding, Hillsdale (NJ), Lawrence Erlbaum.

Sperber D., Wilson D. - (1986) Relevance : Communication and cognition, Oxford, Basic Blackwell (trad. franc., 1989, La pertinence : communication et cognition, Paris, Editions de Minuit).

Townsend D. J. - (1983) Thematic processing in sentences and texts, Cognition, 13, 223-262.

(Accepté le 7 juin 1993.) 